\title{
Routine Prenatal HIV Testing: Women's Concerns and Their Strategies for Addressing Concerns
}

\author{
Pamela Rothpletz-Puglia • Deborah Storm • \\ Carolyn Burr • Deanne Samuels
}

Published online: 8 February 2011

(c) The Author(s) 2011. This article is published with open access at Springerlink.com

\begin{abstract}
The purpose of this exploratory study was to solicit women's opinions about the process of routine prenatal HIV testing to identify strategies for routine testing that will address women's concerns, increase their level of comfort with testing, and support universal prenatal HIV testing. A convenience sample of English-speaking women between 18 and 45 years of age who were HIV-negative or of unknown HIV status were recruited for focus groups at four diverse community sites in four states. Focus group discussion questions addressed health care provider approaches and actions that would make a woman feel more comfortable with the process of routine prenatal HIV testing. Twenty-five women agreed to participate; most women (64\%) were of Black, non-Hispanic race/ethnicity; $44 \%$ were 25-34 years of age. Thematic analysis of women's concerns about routine prenatal HIV testing fell
\end{abstract}

P. Rothpletz-Puglia ( $\square)$

François-Xavier Bagnoud (FXB) Center, School of Nursing (SN), University of Medicine and Dentistry of New Jersey (UMDNJ), 65 Bergen Street, SSB 841, Newark,

NJ 07107, USA

e-mail: rothplpm@umdnj.edu

D. Storm · C. Burr

François-Xavier Bagnoud (FXB) Center, School of Nursing (SN), University of Medicine and Dentistry of New Jersey (UMDNJ), 65 Bergen Street, SSB 8th floor, Newark, NJ 07107, USA

e-mail: stormds@umdnj.edu

C. Burr

e-mail: burrck@umdnj.edu

D. Samuels

Nova Southeastern University, 1750 NE 167 Street, North Miami Beach, FL 33162, USA

e-mail: deanne_samuels@yahoo.com into the following categories: fear, protecting the baby, protecting the woman, confidentiality, and stigma. Women's strategies for addressing these concerns were related to themes of education and information, normalizing HIV testing, patient-provider relationships, systems, and private communication. Participants offered numerous insightful and practical suggestions for addressing their concerns thereby supporting universal routine prenatal HIV testing. The themes that arose in this study support the conclusion that women will be more comfortable with routine prenatal HIV testing if they are fully informed and knowledgeable about the rationale for HIV testing during pregnancy and their right to decline, and if testing is carried out in a confidential and supportive health care environment.

Keywords HIV testing - Prenatal care $\cdot$ HIV prevention

\section{Introduction}

In 2006, the Centers for Disease Control and Prevention (CDC) released revised U.S. Public Health Service (USPHS) recommendations for HIV testing of adults, adolescents, and pregnant women in healthcare settings [1]. These guidelines reiterate 2001 recommendations for universal, routine HIV screening early in pregnancy but advise simplifying the screening process to maximize opportunities for women to learn their HIV status during pregnancy, preserving the woman's option to decline testing, and promoting a patient-provider relationship conducive to optimal clinical and preventive care. The USPHS recommends an opt-out approach during pregnancy where women receive routine HIV testing as part of prenatal care unless they indicate they do not want to be tested and decline. 
Studies have shown that routine prenatal HIV testing is acceptable to women [2-5]. However, evidence suggests that there are ongoing barriers to universal testing related to: (1) individual patient factors, including low perceived risk for HIV, race, socioeconomic status, and confidentiality concerns; (2) provider factors, such as the need to improve knowledge, increase the rates at which they ask about or recommend testing, and strengthen testing practice patterns; and (3) site-related factors, including the need to expand testing in non-traditional settings [6-10]. There is limited information regarding women's specific concerns about prenatal HIV testing and how these concerns can be most effectively addressed [4, 11-13]. The purpose of this study was to solicit women's opinions about the process for routine prenatal HIV testing in order to identify strategies for routine testing that will address women's concerns and support universal prenatal HIV testing.

\section{Methods}

English-speaking women between the ages of 18 and 45 years who were HIV-negative or of unknown HIV status were recruited from four, diverse community sites providing preventive services to women at-risk for HIV infection (Table 1). Case Managers recruited a convenience sample of women from their caseloads. Focus groups were conducted by a single, external moderator at each site over a 3 month time-span. A limited demographic survey including age, race/ethnicity, and pregnancy status was completed prior to discussion.

Focus groups were chosen as the primary means of data collection to gain a deeper understanding of women's opinions about the process of routine prenatal HIV testing. The discussions explored women's thoughts, attitudes, and expectations surrounding testing and allowed investigation of women's perspectives and experiences. Focus group questions addressed health care provider approaches and actions that would potentially make a woman feel more comfortable with the process of routine prenatal HIV testing. Women were asked about the kind of information

Table 1 Community Agencies Providing HIV care and Preventive Services

\begin{tabular}{lll}
\hline Site location & $\begin{array}{l}\text { Number of clients } \\
\text { served }\end{array}$ & $\begin{array}{l}\text { Number of } \\
\text { participants }\end{array}$ \\
\hline Fort Lauderdale, FL & 2,984 & 8 \\
Philadelphia, PA & 2,023 & 4 \\
Dallas, TX & 80 & 5 \\
Milwaukee, WI & 220 & 8 \\
\hline
\end{tabular}

they felt should be provided to make them more comfortable with the process of testing.

Institutional Review Board (IRB) approval was obtained from the principal investigator's university. Participating sites obtained IRB approval or submitted a letter of agreement if the site did not have an IRB. Participants received $\$ 50.00$ compensation. Focus group discussions were audio-taped and transcribed for subsequent content analysis.

\section{Convenience Sample}

Twenty-five women consented to participate. Data were not collected about women who did not choose to participate. About two-thirds (64\%) of participants were of Black, non-Hispanic race/ethnicity, and 44\% were 25-34 years old (see Table 2).

Data Analysis

Transcribed data were entered into the qualitative research software NVivo 7 (QSR International). One coder analyzed the data using content analysis and developed a coding schema using a combination of in vivo terms (literal terms used by the research participants) and open codes (those that were interpreted by the investigator). Data were coded into 21 categories within two primary domains. Some categories also held sub-categories. Once coding was completed, matrices examining variables within and across the two domains were developed to verify saturation of codes and themes.

Table 2 Characteristics of focus group participants

\begin{tabular}{lll}
\hline Characteristic & Number & Percentage \\
\hline Age & 7 & 28 \\
$18-24$ & 11 & 44 \\
$25-34$ & 7 & 28 \\
$35-45$ & & \\
Race/Ethnicity & 4 & 16 \\
White/non hispanic & 16 & 64 \\
Black/non hispanic & 3 & 12 \\
Hispanic & 2 & 8 \\
More than 1 race & & \\
Pregnancy status & - & - \\
Pregnant & 24 & 96 \\
Non-pregnant & 1 & 4 \\
Gave birth within the last year & & \\
\hline
\end{tabular}




\section{Recommendations}

Two major content domains were identified regarding women's opinions about routine prenatal HIV testing: Women's Concerns and Women's Strategies for Addressing Concerns. The 10 most frequently coded categories are described below and listed in Table 3 .

\section{Women's Concerns: Fear}

Women in each focus group expressed concern about the range of fears that women may experience in relation to prenatal HIV testing. This category reflected one of the two most commonly cited categories in the coding schema. Women's fears were sub-categorized into fear of HIV infection, fear of confirming a partner's infidelity, and fear of judgment and/or discharge from medical care if their results are positive.

The most frequently cited fear-related concern was fear of HIV infection which was described in two ways: fear of illness, and more commonly as "a death sentence." Several women in each focus group were unaware of advances in HIV care and treatment, as evidenced by statements such as, "It is pretty much like a death sentence I think, don't you?" or, "I think one of the reasons why people wouldn't rather know is because they live with that on their mind thinking_-'Oh I got AIDS, I'm going to die tomorrow.", Women in each group acknowledged that general knowledge about HIV infection and treatment are lacking, and speculated that a lack of information and education contribute to women's fear about prenatal HIV testing.

Fear of confirming a partner's infidelity also arose as a potential barrier to prenatal HIV testing. Several women in two focus groups discussed this concern. One woman stated, "A lot of people that I know who are married, their husbands cheat on them all the time, every day-you know. They don't want to know. And I think it opens their eyes. If they are HIV-positive, then it's true. It makes it real. You know, I can no longer pretend he's not doing what he is doing. It's real".

The final sentiment about fear was related to medical professionals' perceived judgments and/or fear that a woman would be discharged from medical care if her results are positive. One woman talked about feelings of shame and how a woman might not take the test for fear that the medical doctor would "...be thinking about what she's done in her past." Another woman stated that she was a heroin addict and that in the past she told her medical doctor about her addiction. She reported that the medical doctor promptly called the pharmacy to cancel her prescriptions. She did not relay what the prescriptions were, nor did she understand why the prescriptions were cancelled. Her take-away message was that her medical doctor would no longer take care of her because she admitted to having a heroin addiction. This woman stated that, "I was thinking what about if I had AIDS or HIV, you know, they're going to do the same thing. That was my thinking. You know, I thought that if something (HIV) would happen to me, then he just was going to discharge me from his clinic and that would be it."

\section{Women's Concerns: Protecting Women and Children}

Women in each focus group felt strongly about protecting the unborn baby. This category, along with Fear, was the most frequently mentioned during the focus groups. Almost every participant spoke about maternal instincts to protect babies and children, even in the face of adversity. Though three women felt that there are circumstances in which a woman may not protect her unborn child; teen pregnancy, drug addiction, and depression.

Several women from each focus group were concerned about women feeling coerced or pressured to be tested and spoke of the need to preserve the woman's rights and

Table 3 Content domains and categories, passages coded, and sources

\begin{tabular}{|c|c|c|c|}
\hline Domain & Categories & Number of passages coded & Sources \\
\hline \multirow[t]{5}{*}{ Women's concerns } & Fear & 45 & 4 sites \\
\hline & Protecting the baby & 45 & 4 sites \\
\hline & Protecting the woman & 39 & 4 sites \\
\hline & Confidentiality & 14 & 3 sites \\
\hline & Stigma & 13 & 3 sites \\
\hline \multirow[t]{5}{*}{ Women's strategies for addressing concerns } & Education and information & 125 & 4 sites \\
\hline & Normalizing HIV testing & 43 & 4 sites \\
\hline & Patient-Provider relationship & 39 & 4 sites \\
\hline & Systems & 19 & 3 sites \\
\hline & Private communication & 17 & 3 sites \\
\hline
\end{tabular}


confidentiality. All the women in the focus groups acknowledged these discussions with nods of agreement, or verbally confirmed their agreement.

Several participants expressed concern about whether women would be specifically aware of HIV testing and about how explicit the opt-out process would be. Several participants were concerned about women being tricked or unaware that they could opt-out of HIV testing. For example, one woman stated, "But I think that the doctor should definitely make it clear that they can opt-out. Like you don't want people to feel like after it was done, and you know if there was a positive result, that they were tricked into having it."

Three of the four focus group discussions also included participants' cautions about protecting women by preserving confidentiality in all phases of the routine prenatal HIV testing process. Many participants discussed how HIV testing puts relationships between the woman and her partner at risk and "opens up a whole other can of worms." Some women felt that if the father were in the exam room he may decline the testing or coerce the mother into opting out "for their ego's sake." One woman argued that it is not "safe" to discuss HIV testing with a male partner in the room since many women deal with domestic violence. Another woman felt that "it has to be her choice and it's up to her, how and when the communication takes place with the father."

\section{Women's Concerns: Stigma}

Women from three focus groups mentioned stigma as a barrier to HIV testing. Most of the discussions were related to healthcare providers targeting at-risk women for prenatal HIV testing, thus leaving out a whole segment of the population and stigmatizing urban minorities. Other discussions about stigma involved women's perceptions about healthcare providers' negative attitudes and beliefs about pregnant women living with HIV infection.

Women's Strategies for Addressing Concerns: Information and Normalizing Prenatal HIV Testing

Information and education were the most frequently discussed strategies for enhancing prenatal HIV testing acceptance and for ensuring women's rights. The women in every group felt that general knowledge about HIV infection and treatment are lacking, and they hypothesized that a lack of information contributes to women's fear about prenatal HIV testing. All women felt that people would be more inclined to test for HIV if they were more educated about prevention and treatment.

The content of education that the women felt should be included was information about the risks and benefits of
HIV testing and treatment during pregnancy and beyond. Several women also suggested that information about the confidentiality of test results would help reduce anxiety. Each focus group included a discussion about how HIV has shifted to a chronic disease paradigm. Some women in each group expressed surprise when other women spoke about the efficacy of treatment for reducing perinatal transmission and for preventing disease progression. All women felt that people need more information about HIV infection in this era, as this knowledge may mitigate some of the fear surrounding HIV testing. The women also felt that perinatal transmission statistics would be a powerful educational incentive for perinatal testing, as would information about the impact of treatment on women's health.

Many women also discussed the need for expanding educational efforts beyond targeted high-risk audiences to increase knowledge and awareness at a societal level. Women felt that the provision of all-inclusive HIV prevention and treatment information in high schools or in the community would help people become more comfortable with HIV prevention by normalizing HIV testing. One young woman indicated that she "didn't know crap about AIDS. Kids in the city are getting more information than kids in suburbs. When I moved to Chicago it (HIV) was everywhere, I was like blown away." All of the participants felt that HIV education should be part of mainstream high school education and community programs such as the Special Supplemental Food Program for Women, Infants, and Children (WIC). Some other suggestions for community outreach campaigns and education about HIV prevention and treatment included information booths in malls, outreach teams, Boys and Girls Clubs, and prenatal educational materials.

The women also suggested that information about HIV prevention should be woven into other educational campaigns so that HIV testing is not the sole focal point, but one aspect of preventive health care.

Women's Strategies for Addressing Concerns:

Patent-Provider Relationships, Private

Communications and Systems

Participants spoke about how a comfortable environment and good patient-provider interaction helps to facilitate the process of HIV testing. The barriers to the patient-provider relationship included complaints about physicians not speaking fluent English, caseloads- "a lot of doctors are so tired from other patients," perceptions of healthcare provider attitudes toward women on the margin, and impersonal care. Interestingly, women in a few of the discussions about patient-provider relationships also noted that the 
patient-provider relationship may not be a viable strategy for improving the process of prenatal HIV testing. These women acknowledged that time and caseload barriers are not easily rectified in a world where the budget is bottom line.

Participants agreed that when there is not a strong or effective relationship with a particular physician, there were likely to be other health care professionals and clinical staff members that do relate well to patients, and referred to "Plan B" as capitalizing on the strengths of these individuals. The "Plan B" suggestions included making changes within the system. For example, several women stated that they relate to the person in the front office well. The women suggested that there should be at least one person on staff that women can approach with questions.

The final recommendation that arose in three of the four focus groups was the need for privacy when speaking about HIV prevention, testing, and treatment. Privacy was described as necessary for confidentiality, protecting women, and for providing an environment in which women can trust medical providers. Private communication was also suggested as a way to enable a woman to make a decision without being influenced by her partner, and to protect her from a partner's response if needed.

\section{Discussion}

It is important to acknowledge that the number of focus groups in this study was limited, and that participants were from sites providing services for individuals at risk for HIV. Additional work is needed to identify concerns and strategies about universal routine prenatal HIV testing among women not identified as at risk or in other types of settings.

Despite the exploratory and limited nature of this study, the themes that arose in this study are insightful and useful, and are consistent with other findings in the literature. The provision of information about HIV arose as a way to increase awareness, reduce fear and stigma, and to increase HIV testing acceptance. Studies suggest that prenatal testing rates are lower when women are perceived as low risk by themselves or their providers [2, 14, 15].

Other focus group studies suggest that fear of a positive test result and lack of knowledge regarding the benefits of early detection were common barriers to HIV testing for prenatal patients $[11,13]$. In a survey of men and women about overcoming barriers to HIV testing, knowledge gaps about the benefits of treatment were identified and the authors discuss how people may be more motivated to test if they know the benefits of early treatment [16]. A survey of 748 recently pregnant women showed that prenatal testing was higher among women with knowledge of interventions to prevent perinatal HIV transmission [17]. These findings strongly support the need for increased education and awareness about HIV care and treatment and for providing information-based rationale for prenatal HIV testing.

Suggestions for integrating information into prenatal care included increasing awareness at a societal level rather than targeting certain individuals for testing. Women also felt that HIV testing should be incorporated into other types of prevention efforts so that it is not the sole focal point. Similar to findings from patient focus groups in an inner city urgent care center [18], women in this study suggested media outlets such as television or radio announcements as sources of information about HIV.

Women's suggestions are consistent with a recent call by Arya et al. for media campaigns to address low knowledge about perinatal HIV transmission in the U.S. [19]. Increased public awareness is needed to complement other public health initiatives, such CDC's "One Test Two Lives" campaign which focuses on building capacity among healthcare providers to support universal routine prenatal HIV testing and the prevention of perinatal HIV transmission [20,21].

In addition, effective patient-provider relationships arose as a strategy that would contribute to women's decisions to accept prenatal testing. Studies investigating barriers to universal prenatal testing reported that women's decisions were strongly influenced by providers' recommendations and the patient-provider relationship [7, 22]. Similar to findings from other HIV testing research, women discussed risks of harm related to prenatal HIV testing such as partner violence, confidentiality, and stigma, including fear that health care providers or the community will treat them differently $[8,18,22]$. Strategies for reducing these risks included ensuring private communication and giving information about testing confidentiality.

The themes that arose in this study support the conclusion that women will be more comfortable with universal, routine, prenatal HIV testing if they are fully informed and knowledgeable about the rationale for testing and their right to decline, and if prenatal HIV testing is carried out in a confidential and supportive health care environment.

Acknowledgments This research was funded through Cooperative Agreement \#U69HA05573 with the U.S. Department of Health and Human Services, Health Resources and Services Administration (HRSA), HIV/AIDS Bureau (HAB). The authors would like to thank the Ryan White Care Act sites, and the participants who took the time to help us learn about strategies to improve prenatal HIV testing. The authors would also like to acknowledge the support and wisdom of Linda S. Podhurst, PhD, and HRSA HAB Project Officer Helen Rovito, MS. 
Open Access This article is distributed under the terms of the Creative Commons Attribution Noncommercial License which permits any noncommercial use, distribution, and reproduction in any medium, provided the original author(s) and source are credited.

\section{References}

1. CDC. (2006). Revised recommendations for HIV testing of adults, adolescents, and pregnant women in health care settings. Morbidity and Mortality Weekly Report, 55(No. RR-14), 1-16.

2. Simpson, W. M., Johnstone, F. D., Goldberg, D. J., Gormley, S. M., \& Hart, G. J. (1999). Antenatal HIV testing: Assessment of a routine voluntary approach. British Medical Journal, 318, $1660-1661$.

3. Pearlman, D. N., Averbach, A., Zierler, S., \& Cranston, K. (2005). Disparities in prenatal HIV testing: Evidence for improving implementation of CDC screening guidelines. Journal of the National Medical Association, 97(7), 44S-51S.

4. Podhurst, L. S., Storm, D. S., \& Dolgonos, S. (2009). Women's opinions about routine HIV testing during pregnancy: Implications for the opt-out approach. AIDS Patient Care and STD's, 23(5), 331-337.

5. Chou, R., Smits, A. K., Huffman, L. H., Fo, R., \& Korthuis, R. T. (2005). Prenatal screening for HIV: A review of the evidence for the U.S. Preventive Services Task Force. Annals of Internal Medicine, 143(1), 38-54.

6. Webber, M. (2008). Correlates of prenatal HIV tesing in women with undocumented status at delivery. Maternal and Child Health Journal, 12(4), 427-434.

7. Royce, R. A., Walter, E. B., Fernandez, M. I., Wilson, T. E., Ickovis, J. R., \& Simonds, R. J. (2001). Barriers to universal prenatal HIV testing in 4 US locations in 1997. American Journal of Public Health, 91(5), 727-733.

8. Kelly, K. A., \& Harrison, C. H. (2004). Universal prenatal HIV screening: Patient attitudes and perceptions. Women and Health, 40(1), 41-57. [Abstract].

9. Gray, A. D., Carlson, R., Morgan, M. A., Hawks, D., Schulkin, J., Gray, A. D., et al. (2007). Obstetrician gynecologists' knowledge and practice regarding human immunodeficiency virus screening. Obstetrics and Gynecology, 110(5), 1019-1026. [Research Support, U.S. Gov't, P.H.S.].

10. Burke, R. C., Sepkowitz, K. A., Bernstein, K. T., Karpati, A. M., Myers, J. E., Tsoi, B. W., et al. (2007). Why don't physicians test for HIV? A review of the US literature. AIDS, 21(12), $1617-1624$

11. McKinney, M. M., McSpirit, S., \& Pomeroy, C. (2000). Prenatal HIV prevention practices in a low seroprevalence state. AIDS Education and Prevention, 12(3), 252-262.
12. Lindau, S., Jerome, J., Miller, K., Monk, E., Garcia, P., \& Cohen, M. (2006). Mothers on the margins: Implications for eradicating perinatal HIV. Social Science and Medicine, 62(1), 59-69.

13. de Zuleta, P., \& Boulton, M. (2007). Routine antenatal HIV testing: The responses and perceptions of pregnant women and the viability of informed consent. A qualitative study. Journal of Medical Ethics, 33(6), 329-336.

14. Coleman, V. H., Morgan, M. A., Carlson, R., Hawks, D., \& Schulkin, J. (2009). Patient perceptions of obstetrician-gynecologists' practices related to HIV testing. Maternal and Child Health Journal, 13(3), 355-363.

15. Lawrence, J. M., Liu, I. A., \& Towner, W. J. (2008). Trends and correlates of HIV testing during pregnancy in racially/ethnically diverse insured population, 1997-2006. Maternal and Child Health Journal, 13, 633-640.

16. Spielberg, F., Branson, B. M., Goldbaum, G. M., Lockhart, D., Kurth, A., Celum, C. L., et al. (2003). Overcoming barriers to HIV testing: Preferences for new strategies among clients of a needle exchange, a sexually transmitted disease clinic, and sex venues for men who have sex with men. Journal of Acquired Immune Deficiency Syndrome, 32, 318-328.

17. Anderson, J., \& Sansom, S. (2006). HIV Testing among US women during prenatal care: Findings from the 2002 National Survey of Family Growth. Maternal and Child Health Journal, 10, 413-417.

18. Hutchinson, A. B., Corbie-Smith, G., Thomas, S. B., Mohanan, S., \& del Rio, C. (2004). Understanding the patient's perspective on rapid and routine HIV testing in an inner-city urgent care center. AIDS Education and Prevention, 16(2), 101-114.

19. Arya, M., Levison, J., \& Giordano, T. (2010). Ongoing barriers to HIV testing during pregnancy: A need for media campaigns addressing low knowledge about prenatal HIV transmission among women in the United States. AIDS Patient Care and STD's, 24(2), 71-72.

20. Fowler, M. G., Lampe, M. A., Jamieson, D. J., Kourtis, A. P., Rogers, M. F. (2007). Reducing the risk of mother-to-child human immunodeficiency virus transmission: Past successes, current progress and challenges, and future directions. American Journal of Obstetrics and Gynecology, Supplement, S3-S9. (September).

21. Kriebs, J., \& Shannon, M. (2008). HIV counseling and testing in pregnancy. Journal of Midwifery Women's Health, 53(3), $265-267$.

22. Lindau, S., Jerome, J., Miller, K., Monk, E., Garcia, P., Cohen, M. (2005). Mothers on the margins: Implications for eradicating perinatal HIV. Social Science \& Medicine, 05, 1-11. (in press). [Perinatal HIV transmission; Marginalized Women]. 